\title{
Is Clostridium difficile infection a real threat in patients with ulcerative colitis? A prospective, multicenter study in Korea
}

\author{
Dae Bum Kim ${ }^{1}$, Kang-Moon Lee ${ }^{1}$, Sang Hyoung Park ${ }^{2}$, You Sun Kim, Eun Soo Kim ${ }^{4}$, Jun Lee , \\ Sung-Ae Jung ${ }^{6}$, Geom Seog Seo ${ }^{7}$, Ji Min Lee ${ }^{1}$ \\ ${ }^{1}$ Department of Internal Medicine, St. Vincent's Hospital, College of Medicine, The Catholic University of Korea, Suwon, ${ }^{2}$ Department of \\ Internal Medicine, Asan Medical Center, University of Ulsan College of Medicine, Seoul, ${ }^{3}$ Department of Internal Medicine, Seoul Paik Hospital, \\ Inje University College of Medicine, Seoul, ${ }^{4}$ Department of Internal Medicine, Kyungpook National University Hospital, School of Medicine, \\ Kyungpook National University, Daegu, ${ }^{5}$ Department of Internal Medicine, Chosun University Hospital, Chosun University College of Medicine, \\ Gwangju, ${ }^{6}$ Department of Internal Medicine, Ewha Womans University Medical Center, Ewha Womans University School of Medicine, Seoul, \\ ${ }^{7}$ Department of Internal Medicine, Wonkwang University Hospital, Wonkwang University College of Medicine, Iksan, Korea
}

Background/Aims: Clostridium difficile infection (CDI) has been reported to be a cause of flare-ups in patients with ulcerative colitis (UC). We evaluated the prevalence and clinical outcomes of CDI in patients with UC hospitalized for flare-ups. Methods: This was a prospective, multicenter study including 7 academic teaching hospitals in Korea. All consecutive patients with UC admitted for disease flare-up were enrolled. We detected the presence of CDI by using enzyme immunoassay, realtime polymerase chain reaction (RT-PCR) for toxin genes, and sigmoidoscopy. Results: Eighty-one consecutive patients with UC were enrolled from January 2014 to December 2015. Among 81 patients, 8 (9.9\%) were diagnosed with CDI. Most of the cases were identified by RT-PCR. Enzyme immunoassay was positive in 3 of 8 patients, and only 1 had typical endoscopic findings of pseudomembranous colitis. There were no differences in demographic data, length of hospital stay, or colectomy rate between patients with and without CDI. Conclusions: CDI was not a rare cause of flare-up in patients with UC in Korea. However, CDI did not appear to affect the course of UC flare-up in Korean patients. RT-PCR was sensitive in detecting CDI and can be considered a diagnostic tool in patients with UC flare-up. (Intest Res 2018;16:267-272)

Key Words: Colitis, ulcerative; Clostridium infections; Prevalence; Polymerase chain reaction

\section{INTRODUCTION}

Clostridium difficile is a gram-positive, anaerobic bacterium that produces pathogenic toxins A and B. C. difficile infection (CDI) is the leading cause of nosocomial infectious diarrhea and is associated with substantial morbidity and mortality. The past decade has witnessed a rise in the inci-

Received June 26, 2017. Revised September 25, 2017

Accepted October 19, 2017. Published online January 30, 2018 Correspondence to Kang-Moon Lee, Department of Internal Medicine, St. Vincent's Hospital, College of Medicine, The Catholic University of Korea, 93 Jungbu-daero, Paldal-gu, Suwon 16247, Korea. Tel: +82-31-249-8151, Fax: +82-31-253-8898, E-mail:drmaloman@catholic.ac.kr dence and severity of CDI in Western countries, as well as in Korea. $^{1-4}$

The increased incidence and severity of CDI is even more pronounced in patients with IBD when compared to the general population. ${ }^{5,6}$ A previous study evaluating the incidence of CDI in hospitalized patients in Canada reported that the CDI incidence in patients with IBD was $4.8 \%$, whereas incidence in the general population was $0.45 \%{ }^{7}$ In particular, patients with UC appear to be at higher risk of CDI than those with $\mathrm{CD}^{7.9} \mathrm{C}$. difficile is found in $3 \%$ to $24 \%$ of patients with active UC, and CDI is implicated in 5\% of hospital admissions for UC. ${ }^{5,7,9}$

Furthermore, the presence of CDI in patients with UC is

๑ Copyright 2018. Korean Association for the Study of Intestinal Diseases. All rights reserved.

This is an Open Access article distributed under the terms of the Creative Commons Attribution Non-Commercial License (http://creativecommons.org/licenses/by-nc/4.0)

which permits unrestricted non-commercial use, distribution, and reproduction in any medium, provided the original work is properly cited. 
associated with increased mortality, length of hospital stay, and risk of colectomy. ${ }^{5,10-12}$ Studies across Korea have reported that CDI is associated with a nearly 4 -fold higher risk of acute mortality in hospitalized patients with UC. ${ }^{7}$ Previous studies also have demonstrated increased length of hospital stays and a higher colectomy risk in patients with UC and CDI than in those without CDI. ${ }^{5,11}$ Therefore, detecting CDI early is critical for initiating effective treatment in vulnerable patients, particularly those with UC.

Despite our understanding of the risk of CDI in patients with UC, no data are available to assess the prevalence of CDI in Korean patients with UC. A previous nationwide study in Korea did not find any patients with CDI and comorbid UC. ${ }^{13}$ Therefore, we sought to investigate the prevalence and clinical outcome of CDI in the hospitalized UC patients with flare-up.

\section{METHODS}

\section{Study Design and Subjects}

A prospective, multicenter, observational study was carried out from January 2014 to December 2015 in all consecutive patients with UC admitted because of disease flare-up. All patients had been diagnosed with UC based on clinical, endoscopic, and histological criteria. ${ }^{14}$ Clinical UC disease activity was determined using the Mayo scoring system. ${ }^{15}$ This study was conducted after institutional review board approval from all participating hospitals had been obtained. The informed consent was obtained from all subjects.

\section{Definition and Clinical Outcomes of CDI}

CDI was defined as a positive result on any of the following tests: $C$. difficile toxin A and/or B by enzyme immunoassay (Ridascreen; Biopharm, Darmstadt, Germany); real-time PCR (RT-PCR) of toxin genes; and endoscopic findings, such as multiple whitish or yellowish plaques, consistent with pseudomembranous colitis (PubMed Central [PMC]).

The impact of CDI was evaluated by assessing colectomy rate, inpatient mortality, and length of hospital stay.

\section{Statistical Analysis}

All statistical analyses were performed using SPSS software version 18.0 (SPSS Inc., Chicago, IL, USA). Continuous data were expressed as mean \pm SD and analyzed using independent sample $t$-tests, whereas categorical variables were expressed as numbers and analyzed using the chi-square or Fisher exact test. $P$-values $<0.05$ were considered to be significant.

\section{RESULTS}

\section{Prevalence of CDI and Clinical Characteristics of Patients with and without CDI}

The demographic and clinical characteristics of patients with and without CDI are summarized in Table 1. During the study period, 81 patients were hospitalized because of a UC flare-up. The mean patient age was 39.6 years, and mean disease duration was 3.4 years. The extent of disease was proctitis in 8 patients (9.9\%), left-sided colitis in 23 (28.4\%), and extensive colitis in 50 patients (61.7\%). Twenty-three

Table 1. Demographics and Clinical Characteristics of Patients with and without Clostridium difficile Infection

\begin{tabular}{|c|c|c|c|}
\hline Characteristic & $\begin{array}{c}\text { C. difficile } \\
\text { positive } \\
(n=8)\end{array}$ & $\begin{array}{c}\text { C. difficile } \\
\text { negative } \\
(n=73)\end{array}$ & $P$-value \\
\hline Age (yr) & $45.1 \pm 20.6$ & $38.9 \pm 16.5$ & 0.39 \\
\hline Male sex & $2(25)$ & $38(52)$ & 0.15 \\
\hline Duration of disease (mo) & $29(1-133)$ & $45(0-246)$ & 0.60 \\
\hline Extent of UC $(\%)$ & & & 0.82 \\
\hline Proctitis & 0 & $8(11)$ & \\
\hline Left-sided colitis & $3(38)$ & $20(27)$ & \\
\hline Extensive colitis & $5(63)$ & $45(62)$ & \\
\hline Disease activity (Mayo score) & $9.6 \pm 3.0$ & $9.5 \pm 2.8$ & 0.78 \\
\hline \multicolumn{4}{|l|}{ Medication (\%) } \\
\hline 5-ASA & $8(100)$ & $66(90)$ & 0.40 \\
\hline Corticosteroids & $6(75)$ & $31(42)$ & 0.34 \\
\hline Immunomodulators & $6(75)$ & $30(41)$ & 0.38 \\
\hline Biologics & $4(50)$ & $19(26)$ & 0.18 \\
\hline Proton pump inhibitor & $1(13)$ & $10(14)$ & 0.93 \\
\hline Histamine2 receptor blocker & $1(13)$ & $7(10)$ & 0.79 \\
\hline Probiotics & $2(25)$ & $12(16)$ & 0.54 \\
\hline Charlson comorbidity index & $1.25 \pm 1.17$ & $0.71 \pm 1.15$ & 0.13 \\
\hline White blood cell counts ( $10^{9}$ cells/L) & $8.8 \pm 2.7$ & $9.5 \pm 3.9$ & 0.71 \\
\hline $\mathrm{CRP}(\mathrm{mg} / \mathrm{dL})$ & $4.0 \pm 3.7$ & $4.2 \pm 5.8$ & 0.36 \\
\hline Length of hospital stay (day) & $16.3 \pm 6.3$ & $15.2 \pm 13.9$ & 0.16 \\
\hline Colectomy rate (\%) & 0 & $2(3)$ & 0.64 \\
\hline
\end{tabular}

Values are presented as mean $\pm S D$, number (\%), or median (range). ASA, 5-aminosalicylic acid. 


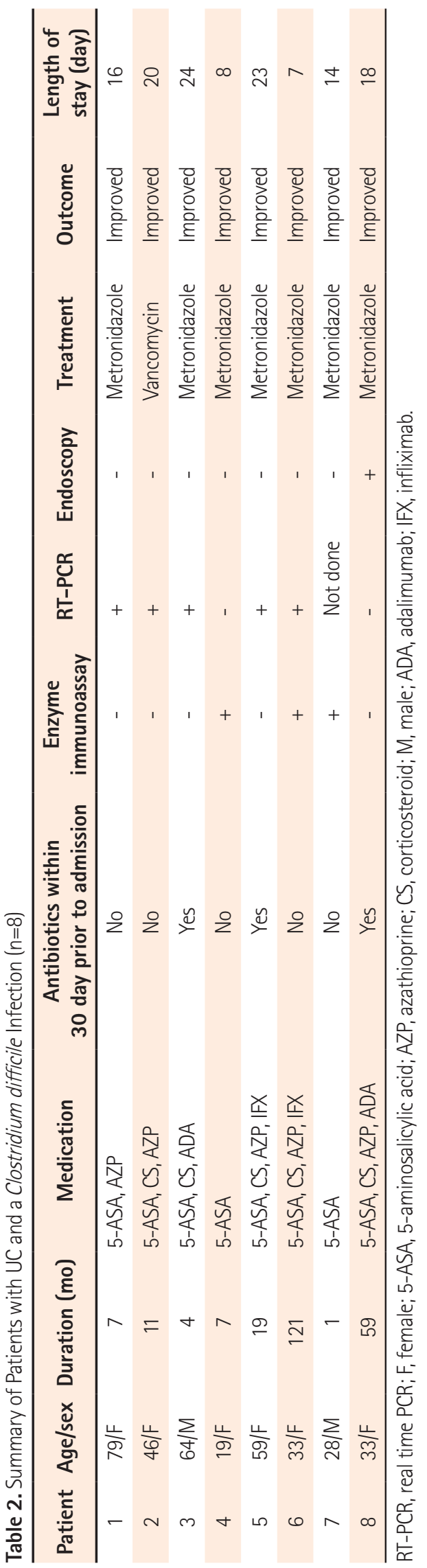

Table 3. Utility of Various Tests for Detecting Clostridium difficile Infection in Patients with UC

\begin{tabular}{lccc}
\hline \multicolumn{1}{c}{ Parameter } & $\begin{array}{c}\text { Enzyme } \\
\text { immunoassay (\%) }\end{array}$ & $\begin{array}{c}\text { RT-PCR } \\
\text { (\%) }\end{array}$ & $\begin{array}{c}\text { Endoscopy } \\
\text { (\%) }\end{array}$ \\
\hline Sensitivity & 37.5 & 71.4 & 12.5 \\
Specificity & 100 & 100 & 100 \\
Positive predictive value & 100 & 100 & 100 \\
Negative predictive value & 93.6 & 96.4 & 91.3 \\
Diagnostic accuracy & 93.8 & 96.7 & 91.4 \\
\hline
\end{tabular}

RT-PCR, real time PCR.

patients (28.4\%) were receiving anti-tumor necrosis factor (anti-TNF) therapy (infliximab or adalimumab) and 45.7\% of patients were receiving systemic corticosteroids. CDI was detected in 8 of the 81 patients (9.9\%) with a UC flare-up. Of the 8 patients with CDI, 6 (75.0\%) were being treated with systemic corticosteroids and $4(50.0 \%)$ were receiving antiTNF therapy. Three patients (37.5\%) had a history of antibiotic use within 30 days. There were no significant differences in age, sex, Charlson comorbidity index and medication history including antibiotics, probiotics, histamine2 receptor blocker and proton pump inhibitor between the 2 groups.

\section{Diagnostic Tests for CDI}

Table 2 shows the diagnostic tests used to detect CDI and the clinical outcomes of patients with both UC and CDI. Among 81 patients, enzyme immunoassay was performed in 81 patients, PCR in 60 patients, and endoscopy was performed in 81 patients. All tests were performed within 3 days after admission. The enzyme immunoassay was positive in 3 of 8 patients, and PCR was positive in 5 of 7 . Four patients had PCR-positive results for stool sample, but a negative enzyme immunoassay result. Endoscopy was performed in all patients with CDI, and only 1 patient demonstrated typical endoscopic findings of PubMed Central. The sensitivity of PCR was higher than that of other methods (Table 3).

\section{Clinical Outcomes of Patients with CDI}

All patients with CDI were treated with oral metronidazole or vancomycin. All patients with CDI who had been admitted for a UC flare-up were discharged after improving clinically, with the mean length of stay being 16 days. No differences in length of hospital stay ( 16.3 days vs. 15.2 days, $P=0.16$ ) was observed between the patients with and without CDI. Two patients without CDI (2.7\%) had a colectomy, whereas no patients with CDI underwent colectomy $(P=0.64)$. 


\section{DISCUSSION}

There is limited data available for the prevalence and clinical outcomes of UC patients with CDI in Asian countries including Korea. To the best of our knowledge, this is the first prospective study evaluating the prevalence and clinical outcomes of CDI in Asian UC patients who have different genetic and ethnic backgrounds, compared to Western countries.

This study detected CDI in 8 of 81 patients (9.9\%) hospitalized with a UC flare-up. Considering the low prevalence of $\mathrm{CDI}$ in the general Korean population, this prevalence rate of CDI in patients with UC is relatively high. ${ }^{1,4,16}$ Our results are in contrast with those of US studies reporting a high incidence of CDI (up to 29\%) among hospitalized patients with IBD, but are similar to results of studies from European countries (3.6\%-7.0\%). ${ }^{17-20}$ Possible explanations for this discrepancy include differences in microbiological techniques, and heterogeneity of the patient populations studied. In addition, many recent studies were retrospective in nature and used diagnostic codes, where miscoding could either underestimate or overestimate CDI prevalence. In addition, the availability of information could have been limited, as CDI might only have been assessed in selected patient groups for clinical reasons. ${ }^{7,8,10,11}$

Some previous studies have reported that CDI in patients with IBD may be associated with older age, steroid use, disease severity, and recent antibiotic therapy., ${ }^{5,21}$ However, in this study, of the 8 patients with UC and CDI, only 3 (37.5\%) were diagnosed with CDI while on antibiotic therapy. This suggests that antibiotics may play a less significant role in the development of CDI in patients with UC than in the general population, which is consistent with some other studies. ${ }^{6} \mathrm{~A}$ retrospective study showed that $40 \%$ of CDI patients with IBD used antibiotics, whereas $69 \%$ of CDI patients without IBD used antibiotics. ${ }^{6}$ A recent prospective study demonstrated that CDI is not associated with antibiotic use. ${ }^{22}$ However, given the small sample size of patients with CDI, our findings should be interpreted with caution.

Several studies in Western countries have reported that the presence of CDI in patients with UC is associated with increased mortality, length of hospital stay, and colectomy risk. ${ }^{5,10-12}$ A recent study in the United States reported increased mortality and colectomy risk in patients with CDI, particularly if they had UC as well. ${ }^{11}$ Similarly, in Canada, CDI worsened the clinical outcomes of UC by increasing length of hospital stay and colectomy rate. ${ }^{23}$ However, in contrast with these results from Western countries, CDI did not affect the clinical course of hospitalized Korean patients with UC in our study. Similarly, the severity of CDI in the general Korean population is lower than that in Western countries, which may be because of lower prevalence of a hypervirulent strain, compared with that in Western countries. ${ }^{1,13}$ However, $C$. difficile ribotype 027, which is the hypervirulent strain, was reported for the first time in Korea in 2009. ${ }^{24}$ Therefore, close attention to the increased prevalence and severity of CDI is needed, particularly in patients with risk factors, such as UC.

Owing to their shared symptoms, it is often difficult to distinguish CDI from an IBD exacerbation. There is no testing strategy is optimally sensitive and specific for diagnosing CDI in patients with UC, and no study has evaluated the diagnostic accuracy of CDI in patients with IBD. Toxin enzyme immunoassay is the most widely used diagnostic test because of the ease of use and objective interpretation. Endoscopy is also performed to assess disease activity or to detect pseudomembranes in patients with a UC flare-up when CDI is suspected. However, enzyme immunoassays have low sensitivity (60\%-70\%), suggesting that a missed diagnosis is possible. ${ }^{25,26}$ In addition, pseudomembranes are infrequently seen in UC patients with CDI. ${ }^{5,27}$ Previous studies demonstrated that only $9 \%$ of patients with IBD have pseudomembranes on sigmoidoscopy or colonoscopy. ${ }^{28}$ Indeed, in our study, among the 4 PCR-positive patients, 3 (75\%) had a negative enzyme immunoassay result, and only 1 had pseudomembranes on sigmoidoscopy. Therefore, PCR may offer an advantage over enzyme immunoassay or endoscopy in diagnosing CDI in patients with UC. However, positive PCR results should be interpreted with caution, given the possibility of false positive PCR results.

One of this study's strengths was that consecutive patients were enrolled prospectively. Most previous studies used retrospectively collected cases derived from diagnostic codes instead of microbiological tests. In addition, we performed PCR in most cases, which is the most sensitive method for diagnosing CDI, whereas previous studies diagnosed CDI by using toxin assay with a low sensitivity. Therefore, in this study, the overall prevalence of CDI in hospitalized patients with UC was relatively more precise.

There were several limitations to this study. First, the number of patients included was small. Second, only academic teaching hospitals participated in this study, and the subjects attending these hospitals tend to be more severely ill than patients from smaller hospitals. In addition, it is uncertain that patients with positive PCR results have CDI or are simply colonized. Therefore, further studies are warranted to 
determine whether patients with negative enzyme immunoassay results and positive PCR results should be treated or not. However, despite these limitations, this prospective study provides important data on the prevalence and clinical impact of CDI in Korean patients with a UC flare-up.

In conclusion, the prevalence of CDI in patients with a UC flare-up was not lower in Korea, compared to that in Western countries. Therefore, CDI should be checked in patients with a UC flare-up. ${ }^{29}$ RT-PCR was the most sensitive assay method and should be considered a diagnostic test for CDI in patients with UC. ${ }^{30}$

\section{FINANCIAL SUPPORT}

The authors received no financial support for the research, authorship, and/or publication of this article.

\section{CONFLICT OF INTEREST}

No potential conflict of interest relevant to this article was reported.

\section{AUTHOR CONTRIBUTION}

K.M.L. conceived and designed the study. D.B.K. and J.M.L. analyzed the data. D.B.K., S.H.P., Y.S.K., E.S.K., J.L., S.A.J., and G.S.S. collected the data. D.B.K. wrote the manuscript. All authors contributed to the editing, reviewing, and final approval of the manuscript.

\section{REFERENCES}

1. Kim YS, Han DS, Kim YH, et al. Incidence and clinical features of Clostridium difficile infection in Korea: a nationwide study. Epidemiol Infect 2013;141:189-194.

2. Ananthakrishnan AN. Clostridium difficile infection: epidemiology, risk factors and management. Nat Rev Gastroenterol Hepatol 2011;8:17-26.

3. Bassetti M, Villa G, Pecori D, Arzese A, Wilcox M. Epidemiology, diagnosis and treatment of Clostridium difficile infection. Expert Rev Anti Infect Ther 2012;10:1405-1423.

4. Choi HY, Park SY, Kim YA, et al. The epidemiology and economic burden of Clostridium difficile infection in Korea. Biomed Res Int 2015;2015:510386. doi: 10.1155/2015/510386.

5. Issa M, Vijayapal A, Graham MB, et al. Impact of Clostridium difficile on inflammatory bowel disease. Clin Gastroenterol Hepatol 2007;5:345-351.
6. Bossuyt P, Verhaegen J, Van Assche G, Rutgeerts P, Vermeire S. Increasing incidence of Clostridium difficile-associated diarrhea in inflammatory bowel disease. J Crohns Colitis 2009;3:4-7.

7. Nguyen GC, Kaplan GG, Harris ML, Brant SR. A national survey of the prevalence and impact of Clostridium difficile infection among hospitalized inflammatory bowel disease patients. Am J Gastroenterol 2008;103:1443-1450.

8. Ricciardi R, Ogilvie JW Jr, Roberts PL, Marcello PW, Concannon TW, Baxter NN. Epidemiology of Clostridium difficile colitis in hospitalized patients with inflammatory bowel diseases. Dis Colon Rectum 2009;52:40-45.

9. Rodemann JF, Dubberke ER, Reske KA, Seo DH, Stone CD. Incidence of Clostridium difficile infection in inflammatory bowel disease. Clin Gastroenterol Hepatol 2007;5:339-344.

10. Ananthakrishnan AN, McGinley EL, Binion DG. Excess hospitalisation burden associated with Clostridium difficile in patients with inflammatory bowel disease. Gut 2008;57:205-210.

11. Ananthakrishnan AN, McGinley EL, Saeian K, Binion DG. Temporal trends in disease outcomes related to Clostridium difficile infection in patients with inflammatory bowel disease. Inflamm Bowel Dis 2011;17:976-983.

12. Jen MH, Saxena S, Bottle A, Aylin P, Pollok RC. Increased health burden associated with Clostridium difficile diarrhoea in patients with inflammatory bowel disease. Aliment Pharmacol Ther 2011;33:1322-1331.

13. Han SH, Kim H, Lee K, et al. Epidemiology and clinical features of toxigenic culture-confirmed hospital-onset Clostridium difficile infection: a multicentre prospective study in tertiary hospitals of South Korea. J Med Microbiol 2014;63(Pt 11):1542-1551.

14. Choi CH, Jung SA, Lee BI, et al. Diagnostic guideline of ulcerative colitis. Korean J Gastroenterol 2009;53:145-160.

15. Schroeder KW, Tremaine WJ, Ilstrup DM. Coated oral 5-aminosalicylic acid therapy for mildly to moderately active ulcerative colitis. A randomized study. N Engl J Med 1987;317:1625-1629.

16. Kim J, Pai H, Seo MR, Kang JO. Epidemiology and clinical characteristics of Clostridium difficile infection in a Korean tertiary hospital. J Korean Med Sci 2011;26:1258-1264.

17. Markowitz JE, Brown KA, Mamula P, Drott HR, Piccoli DA, Baldassano RN. Failure of single-toxin assays to detect clostridium difficile infection in pediatric inflammatory bowel disease. Am J Gastroenterol 2001;96:2688-2690.

18. Regnault H, Bourrier A, Lalande V, et al. Prevalence and risk factors of Clostridium difficile infection in patients hospitalized for flare of inflammatory bowel disease: a retrospective assessment. Dig Liver Dis 2014;46:1086-1092. 
19. Masclee GM, Penders J, Jonkers DM, Wolffs PF, Pierik MJ. Is clostridium difficile associated with relapse of inflammatory bowel disease? Results from a retrospective and prospective cohort study in the Netherlands. Inflamm Bowel Dis 2013;19:2125-2131.

20. Ott C, Girlich C, Klebl F, et al. Low risk of Clostridium difficile infections in hospitalized patients with inflammatory bowel disease in a German tertiary referral center. Digestion 2011;84:187192.

21. Sinh P, Barrett TA, Yun L. Clostridium difficile infection and inflammatory bowel disease: a review. Gastroenterol Res Pract 2011;2011:136064. doi: 10.1155/2011/136064.

22. Roy A, Lichtiger S. Clostridium difficile Infection: a rarity in patients receiving chronic antibiotic treatment for Crohn's disease. Inflamm Bowel Dis 2016;22:648-653.

23. Murthy SK, Steinhart AH, Tinmouth J, Austin PC, Daneman $\mathrm{N}$, Nguyen GC. Impact of Clostridium difficile colitis on 5-year health outcomes in patients with ulcerative colitis. Aliment Pharmacol Ther 2012;36:1032-1039.
24. Tae CH, Jung SA, Song HJ, et al. The first case of antibiotic-associated colitis by Clostridium difficile PCR ribotype 027 in Korea. J Korean Med Sci 2009;24:520-524.

25. Issa M, Ananthakrishnan AN, Binion DG. Clostridium difficile and inflammatory bowel disease. Inflamm Bowel Dis 2008;14:1432-1442.

26. Bartlett JG, Gerding DN. Clinical recognition and diagnosis of Clostridium difficile infection. Clin Infect Dis 2008;46 Suppl 1:S12-S18.

27. Ben-Horin S, Margalit M, Bossuyt P, et al. Prevalence and clinical impact of endoscopic pseudomembranes in patients with inflammatory bowel disease and Clostridium difficile infection. J Crohns Colitis 2010;4:194-198.

28. Goodhand JR, Alazawi W, Rampton DS. Systematic review: Clostridium difficile and inflammatory bowel disease. Aliment Pharmacol Ther 2011;33:428-441.

29. Choi CH, Moon W, Kim YS, et al. Second Korean guidelines for the management of ulcerative colitis. Intest Res 2017;15:7-37.

30. Song PH, Min JH, Kim YS, et al. Rapid and accurate diagnosis of Clostridium difficile infection by real-time polymerase chain reaction. Intest Res 2018;16:109-115. 